\title{
II. (CCXXVI.) Einmündung der Vena pulmonalis dextra superior in die Vena cava superior.
}

2. Fall eigener Beobachtung und 4. sicherer Fall aller Beobachtungen.

Aus der Zusammenstellung der bis 1876 reichenden Literatur über diese Varietät in der Mittheilung ${ }^{1}$ ) und Abbildung des 1. Falles eigener Beobachtung dieser Varietät geht hervor, „dass von übrigens wohlgebildeten Individuen J. Fr. Meckel²) den so sicheren Fall abgebildet hat und dass ich, 56 Jahre später, den 2. Fall beschrieben und abgebildet habe.

Später, d. i. 4 Jahre nach der Veröffentlichung meines ersten Falles, gab C. Gegenbaur ${ }^{3}$ ) über einen 3. Fall, der dem Falle von Meckel ähnlich ist, eine Beschreibung mit Abbildung.

Diesen Fällen kann ich folgenden 4. Fall (2. Fall eigener Beobachtung) ăreihen, der an der zu den Präparirübungen abgelassenen, äusserlich wohlgebildeten Leiche eines alten Weibes am 12. December 1884, also 4 Jahre nach der Veröffentlichung des Gegenbaur'schen Falles, zur Beobachtung kam.

Die Trachea und die Bronchi verhalten sich normal.

Die rechte Lunge besitzt 3 , die linke 2 Lippen. Beide Lungen sind völlig tuberculös und jede ist an der Spitze mit einer grossen Caverne behaftet.

Das Herz ist von gewöhnlicher Gestalt und Grösse und anch pathologisch nicht verändert. Auch weist sein Atrium sinistrum 4 Ostia venarum pulmonalium, wie in der Norm, auf, aber das diesmal ungewöhnlich kleine Ostium dextrum anterius superius gehört nicht der Vena pulmonalis dextra superior an. Der Arcus aortae und seine grossen Stämme, die Arteria pulmonalis communis, deren Aeste und das Ligamentum arteriosum, die Vena cava inferior, die Venae azygae und die beiden Venae pulmonales sinistrae verhalten sich wie gewöhnlich.

Fine Ausnahme aber machen die Vena cava superior und die Venae pulmonales dextrae.

Die Vena cava superior nimmt nehmlich die Vena pulmonalis dextra superior an ihrer rechten Seitenwand, gleich unter der Einsenkung der Vena

1) Ein Fall von Einmündung der Vena pulınonalis dextra superior in die Vena cava superior. Dieses Archiv Bd. 68. Berlin 1876. Meine anat. Notizen No. LXXI. S. 284. Taf. V. Fig. 3.

2) Tab. anat.-pathol. Fasc. II. Lipsiae 1820. Tab. IX. Fig. 2 d.

3) Ein Fall von Einmündung der oberen rechten Lungenvene in die obere Hohlvene. Morphol. Jahrb. Bd. VI. Leipzig 1880. S. 315. Mit Holzschn. 
azygos an der hinteren Wand und $2,4 \mathrm{~cm}$ oberhalb des Atrium dextrum anf; statt 2 Venae pulmonales dextrae giebt es deren 3.

Die Vena cava superior ist $7,8 \mathrm{~cm}$ lang, wovon $3,4 \mathrm{~cm}$ auf das Stück äber der Einmündung der Vena azygos, $7 \mathrm{~mm}$, auf die Einmündungsstelle der letzteren, $1,3 \mathrm{~cm}$ auf die Finmündungsstelle der Vena pulmonalis dextra superior nnd 2,4 $\mathrm{cm}$ auf das Endstück zwischen der Einmündung dieser Vene und dem Atrium dextrum kommen. Sie ist oberhalb der Einmündung der Vena azygos $1,8 \mathrm{~cm}$, unter der Einmündung der Vena pulmonalis dextra superior $2 \mathrm{~cm}$ weit. An dem $4 \mathrm{~cm}$ langen Endstücke von der Einsenkung der Vena azygos abwärts ist dieselbe mit dem serösen Blatte des Pericardium bekleidet.

Von den 3 Venae pulmonales dextrae konmt die superior aus dem oberen, die media aus dem mittleren und die inferior aus dem unteren Lappen der rechten Lunge. Davon ist die inferior die stärkste und die media die schwächste.

Die Vena pulmonalis dextra superior taucht in oberen Ende des Bilus pulmonalis aus dem oberen Lappen mit 2 Wurzeln üher und vor dem oberen secundären Aste des oberen primitiven Astes der Arteria pulmonalis dextra auf. Nach Vereinigung der Wurzeln zu einem Stamm verläuft dieser vor dem oberen primitiven Ast der Arteria pulmonalis dextra, ihn etwas schräg kreuzend, quer.medianwärts, um an der angegebenen Stelle der Vena cava superior in diese zu münden. Die obere Wurzel ist $4 \mathrm{~mm}$, die untere $8 \mathrm{~mm}$, der Stamm $4 \mathrm{~cm}$, also die Vene oben $1,4 \mathrm{~cm}$, unten $1,8 \mathrm{~cm}$ lang. Die abere Wurzel ist $7 \mathrm{~mm}$, die untere $5 \mathrm{~mm}$ und der Stamm 12 bis $13 \mathrm{~mm}$ weit. Sie ist im grösseren Umfange mit dem serösen Blatte des Pericardium bekleidet, liegt also im Pericardialsacke. Sie besitzt keine Klappe.

Während die Vena pulmonalis dextra superior in die Vena cava superior mündet und ihr Blut durch diese in das Atrium dextrum geführt hat, münden die V. p. dextra media und inferior in das Atrium sinistrum und zwar erstere am Ostium pulmonale dextrum anterius superius, letztere am $0 . p . d$. posterius inferius, wie die sonst aus der ganzen rechten Lage kommenden beiden Venae pulmonales dextra der Norm.

Das Präparat habe ich in meiner Sammlung an der medicinischen Akademie in St. Petersburg deponirt.

Mein 2. Fall ist meinem 1. Falle ähnlich. In beiden Fällen gab es 3 Venae pulmonales dextrae, wovon die Superior in die Vena cava superior, die Media und Inferior durch besondere Ostia in das Atrium sinistrum mündeten. Im 2. Falle waren 2 Venae pulmonales sinistrae zugegen, während im 1. Falle nur eine Vena pulmonalis sinistra unica existirte. Die Fälle von Meckel und Gegenbaur glichen einander: in beiden gab es nur 2 Venae pulmonales dextrae. Meckel's Fall and mein 
1. Fall hat männlichen Individuen, mein 2. Fall einem weiblichen Individuum angehört. Welchem Geschlechte in Gegenbaur's Falle das Individuum gehörte, ist nicht angegeben.

III. (CCXXVII.) Verästelung des Ramus superficialis des Nervus radialis in der Haut des Rückens der ganzen Hand und aller Finger bei nur verkümmert vorhandenen Ramus dorsalis des Nervus ulnaris.

2. Fall eigener Beobachtnng.

Kaufmann, Turner und Giacomini haben je einen Fall mitgetheilt, in welchem der Ramus superficialis des Nervus radialis alle dorsalen Fingernerven abgab und den Ramus dorsalis des Nervus ulnaris ersetzte ${ }^{3}$ ). I Ich $^{2}$ ) habe einen Fall eigener Beobachtung erwähnt, in welchem derselbe Nerv ebenfalls in der Haut des Rückens aller Finger sich verzweigte, aber doch der Ramus dorsalis des Nervus ulnaris mit Verzweigung in der Haut des Rückens des 4 . und $\overline{5}$. Fingers zugleich vorhanden war.

F. A. Longet ${ }^{3}$ ) hat einen Fall beobachtet, in welchem derselbe Nerv alle dorsalen Fingernerven, mit Ausnahme des $\mathbf{N}$. dorsalis ulnaris dig. quinti, der vom Ramus dorsalis des $\mathrm{N}$. ulnaris kam, abgab.

Einen solchen Fall hat an der linken Hand eines Mannes auch $\mathrm{Ozenne}{ }^{4}$ ) beobachtet und einen gleichen bei seinem Collegen Verhère gesehen.

Ich kann diesen Fällen folgenden zweiten Fall eigener Beobachtung zugesellen, in welchem der Ramus superficialis des Nervus radialis wieder für alle Finger dorsale Hautnerven lieferte, anch der Ramus dorsalis des Nervus ulnaris nicht ganz fehlte, aber nur als $\mathrm{N}$. digitalis dorsalis ulnaris für den 5 . Finger existirte, nachdem er einen $Z$ weig vom Nervus cutaneus brachii medius aufgenommen hatte.

1) Bei J. Henle, Nervenlehre. Braunschweig 1871. S. 499.

3) Ungewöhnliche Anordnung der Hautnerven am Handrücken. Vermischte Notizen. No.X. Dieses Archiv Bd. 54. 1872. S. 180.

3) Anatomie et Physiologie du système nerveux. Tom. I. Paris 1842. p. 858 et 863 . Note.

4) Bull, de la Société anatomique de Paris. 1883. p. 108. 